TRANSACTIONS OF THE

AMERICAN MATHEMATICAL SOCIETY

Volume 360, Number 11, November 2008, Pages 6031-6043

S 0002-9947(08)04485-1

Article electronically published on June 26, 2008

\title{
GAUSSIAN ESTIMATES FOR FUNDAMENTAL SOLUTIONS OF SECOND ORDER PARABOLIC SYSTEMS WITH TIME-INDEPENDENT COEFFICIENTS
}

\author{
SEICK KIM
}

\begin{abstract}
Auscher, McIntosh and Tchamitchian studied the heat kernels of second order elliptic operators in divergence form with complex bounded measurable coefficients on $\mathbb{R}^{n}$. In particular, in the case when $n=2$ they obtained Gaussian upper bound estimates for the heat kernel without imposing further assumption on the coefficients. We study the fundamental solutions of the systems of second order parabolic equations in the divergence form with bounded, measurable, time-independent coefficients, and extend their results to the systems of parabolic equations.
\end{abstract}

\section{INTRODUCTION}

In 1967, Aronson [1] proved Gaussian upper and lower bounds for the fundamental solutions of parabolic equations in divergence form with bounded measurable coefficients. To establish the Gaussian lower bound Aronson made use of the Harnack inequality for nonnegative solutions which was proved by Moser in 1964 (see [17). Related to Moser's parabolic Harnack inequality, we should mention Nash's earlier paper [18] where the Hölder continuity of weak solutions to parabolic equations in divergence form was established. In 1985, Fabes and Stroock 10 showed that the idea of Nash could be used to establish a Gaussian upper and lower bound on the fundamental solution. They showed that actually such Gaussian estimates could be used to prove Moser's Harnack inequality. We note that Aronson also obtained Gaussian upper bound estimates of the fundamental solution without using Moser's Harnack inequality.

In [2, Auscher proposed a new proof of Aronson's Gaussian upper bound estimates for the fundamental solution of second order parabolic equations with timeindependent coefficients. His method relies crucially on the assumption that the coefficients are time-independent, and thus it does not exactly reproduce Aronson's result, which is valid even for the time-dependent coefficients case. However, his method is interesting in the sense that it carries over to equations with complex coefficients provided that the complex coefficients are a small perturbation of real coefficients. Along with this direction, Auscher, McIntosh and Tchamitchian also showed that the heat kernel of second order elliptic operators in divergence form with complex bounded measurable coefficients in the two dimensional space has a Gaussian upper bound (see [3] and also [5]).

Received by the editors April 20, 2005 and, in revised form, November 3, 2006.

2000 Mathematics Subject Classification. Primary 35A08, 35B45; Secondary 35K40.

Key words and phrases. Gaussian estimates, a priori estimates, parabolic system.

(C)2008 American Mathematical Society Reverts to public domain 28 years from publication 
We would like to point out that a parabolic equation with complex coefficients is, in fact, a special case of a system of parabolic equations. From this point of view, Hofmann and the author showed that the fundamental solution of a parabolic system has an upper Gaussian bound if the system is a small perturbation of a diagonal system, which, in particular, generalized the result of Auscher mentioned above to the time-dependent coefficients case (see [12]). However, the above mentioned result of Auscher, McIntosh and Tchamitchian regarding the heat kernel of two dimensional elliptic operators with complex coefficients does not follow directly from our result.

One of the main goals of this article is to provide a proof that weak solutions of the parabolic system of divergence type with time-independent coefficients associated to an elliptic system in two dimensions enjoy the parabolic local boundedness property and to show that its fundamental solution has a Gaussian upper bound. More generally, we show that if weak solutions of an elliptic system satisfy Hölder estimates at every scale, then weak solutions of the corresponding parabolic system with time-independent coefficients also satisfy similar parabolic Hölder estimates from which, in particular, the parabolic local boundedness property follows easily. Also, such an argument allows one to derive Hölder continuity estimates for weak solutions of parabolic equations with time-independent coefficients directly from De Giorgi's theorem [7] on elliptic equations, bypassing Moser's parabolic Harnack inequality. In fact, this is what Auscher really proved in the setting of complex coefficients equations by using a functional calculus method (see [2] and also [4, [5]). Even in those complex coefficients settings, we believe that our approach is much more straightforward and thus appeals to a wider readership.

Finally, we would like to point out that in this article, we are mainly interested in global estimates and that we do not attempt to treat, for example, the systems with lower order terms, etc. However, let us also mention that, with some extra technical details, our methods carry over to those cases as well as to the systems of higher order; see e.g., [4, [5] for the details, and also Remark 3.5.

The remaining sections are organized in the following way. In Section 2 we give notation, definitions, and some known facts. We state the main results in Section 3 and give the proofs in Section 4 .

\section{Notation AND DEFINITIONS}

\subsection{Geometric notation.}

(1) $\mathbb{R}^{n}=n$-dimensional real Euclidean space.

(2) $x=\left(x_{1}, \ldots, x_{n}\right)$ is an arbitrary point of $\mathbb{R}^{n}$.

(3) $X=(x, t)$ denotes an arbitrary point in $\mathbb{R}^{n+1}$, where $x \in \mathbb{R}^{n}$ and $t \in \mathbb{R}$.

(4) $B_{r}(x)=\left\{y \in \mathbb{R}^{n}:|y-x|<r\right\}$ is an open ball in $\mathbb{R}^{n}$ with center $x$ and radius $r>0$. We sometimes drop the reference point $x$ and write $B_{r}$ for $B_{r}(x)$ if there is no danger of confusion.

(5) $Q_{r}(X)=\left\{(y, s) \in \mathbb{R}^{n+1}:|y-x|<r\right.$ and $\left.t-r^{2}<s<t\right\}$. We sometimes drop the reference point $X$ and write $Q_{r}$ for $Q_{r}(X)$.

(6) $Q_{r}^{*}(X)=\left\{(y, s) \in \mathbb{R}^{n+1}:|y-x|<r\right.$ and $\left.t<s<t+r^{2}\right\}$.

(7) $Q_{r, s}(X)=\left\{(y, s) \in Q_{r}(X)\right\}$; i.e., $Q_{r, s}(X)=B_{r}(x) \times\{s\}$ if $s \in\left(t-r^{2}, t\right)$ and $Q_{r, s}(X)=\emptyset$ otherwise. We sometimes drop the reference point $X$ and write $Q_{r, s}$ for $Q_{r, s}(X)$. 
(8) For a cylinder $Q=\Omega \times(a, b) \subset \mathbb{R}^{n+1}, \partial_{P} Q$ denotes its parabolic boundary, namely, $\partial_{P} Q=\partial \Omega \times(a, b) \cup \bar{\Omega} \times\{a\}$, where $\partial \Omega$ is the usual topological boundary of $\Omega \subset \mathbb{R}^{n}$ and $\bar{\Omega}$ is its closure.

\subsection{Notation for functions and their derivatives.}

(1) For a mapping from $\Omega \subset \mathbb{R}^{n}$ to $\mathbb{R}^{N}$, we write $\boldsymbol{f}(x)=\left(f^{1}(x), \ldots, f^{N}(x)\right)^{T}$ as a column vector.

(2) $\bar{f}_{Q}=\frac{1}{|Q|} \int_{Q} f$, where $|Q|$ denotes the volume of $Q$.

(3) $u_{t}=\partial u / \partial t$.

(4) $D_{x_{i}} u=D_{i} u=u_{x_{i}}=\partial u / \partial x_{i}$.

(5) $D u=\left(u_{x_{1}}, \ldots, u_{x_{n}}\right)^{T}$ is the spatial gradient of $u=u(x, t)$.

(6) For $\boldsymbol{f}=\left(f^{1}, \ldots, f^{N}\right)^{T}, D \boldsymbol{f}=\left(D f^{1}, \ldots, D f^{N}\right)$; that is, $D \boldsymbol{f}$ is the $n \times N$ matrix whose $i$-th column is $D f^{i}$.

\subsection{Function spaces.}

(1) For $\Omega \subset \mathbb{R}^{n}$ and $p \geq 1, L^{p}(\Omega)$ denotes the space of functions with the following norms:

$$
\|u\|_{L^{p}(\Omega)}=\left(\int_{\Omega}|u(x)|^{p} d x\right)^{1 / p} \quad \text { and } \quad\|u\|_{L^{\infty}(\Omega)}=\operatorname{ess}_{\sup _{\Omega}}|u| .
$$

(2) $C^{\mu}(\Omega)$ denotes the space of functions that are Hölder continuous with the exponent $\mu \in(0,1]$, and

$$
[u]_{C^{\mu}(\Omega)}=\sup _{x \neq x^{\prime} \in \Omega} \frac{\left|u(x)-u\left(x^{\prime}\right)\right|}{\left|x-x^{\prime}\right|^{\mu}}<\infty .
$$

(3) The Morrey space $M^{2, \mu}(\Omega)$ is the set of all functions $u \in L^{2}(\Omega)$ such that

$$
\|u\|_{M^{2, \mu}(\Omega)}=\sup _{B_{\rho}(x) \subset \Omega}\left(\rho^{-\mu} \int_{B_{\rho}(x)}|u|^{2}\right)^{1 / 2}<\infty .
$$

(4) $C_{P}^{\mu}(Q)$ denotes the space of functions defined on $Q \subset \mathbb{R}^{n+1}$ such that

$$
[u]_{C_{P}^{\mu}(Q)}=\sup _{X \neq X^{\prime} \in Q} \frac{\left|u(X)-u\left(X^{\prime}\right)\right|}{d_{P}\left(X, X^{\prime}\right)^{\mu}}<\infty,
$$

where $d_{P}\left(X, X^{\prime}\right)=\max \left(\left|x-x^{\prime}\right|, \sqrt{\left|t-t^{\prime}\right|}\right)$.

\subsection{Elliptic and parabolic systems and their adjoints.}

Definition 2.1. We say that the coefficients $A_{i j}^{\alpha \beta}(x)$ satisfy the uniform ellipticity condition if there exist numbers $\nu_{0}, M_{0}>0$ such that for all $x \in \mathbb{R}^{n}$ we have

$$
\left\langle\boldsymbol{A}^{\alpha \beta}(x) \boldsymbol{\xi}_{\beta}, \boldsymbol{\xi}_{\alpha}\right\rangle \geq \nu_{0}|\boldsymbol{\xi}|^{2} \quad \text { and } \quad\left|\left\langle\boldsymbol{A}^{\alpha \beta}(x) \boldsymbol{\xi}_{\beta}, \boldsymbol{\eta}_{\alpha}\right\rangle\right| \leq M_{0}|\boldsymbol{\xi}||\boldsymbol{\eta}|
$$

where we use the following notation:

(1) For $\alpha, \beta=1, \ldots, n, \boldsymbol{A}^{\alpha \beta}(x)$ are $N \times N$ matrices with $(i, j)$-entries $A_{i j}^{\alpha \beta}(x)$.

(2) $\boldsymbol{\xi}_{\alpha}=\left(\xi_{\alpha}^{1}, \ldots, \xi_{\alpha}^{N}\right)^{T}$ and $|\boldsymbol{\xi}|^{2}=\sum_{\alpha=1}^{n} \sum_{i=1}^{N}\left|\xi_{\alpha}^{i}\right|^{2}$.

(3) $\left\langle\boldsymbol{A}^{\alpha \beta}(x) \boldsymbol{\xi}_{\beta}, \boldsymbol{\eta}_{\alpha}\right\rangle=\sum_{\alpha, \beta=1}^{n} \sum_{i, j=1}^{N} A_{i j}^{\alpha \beta}(x) \xi_{\beta}^{j} \eta_{\alpha}^{i}$.

We emphasize that we do not assume that the coefficients are symmetric. 
Definition 2.2. We say that a system of $N$ equations on $\mathbb{R}^{n}$

$$
\sum_{j=1}^{N} \sum_{\alpha, \beta=1}^{n} D_{x_{\alpha}}\left(A_{i j}^{\alpha \beta}(x) D_{x_{\beta}} u^{j}\right)=0 \quad(i=1, \ldots, N)
$$

is elliptic if the coefficients satisfy the uniform ellipticity condition. We often write the above system in a vector form:

$$
L \boldsymbol{u}:=\sum_{\alpha, \beta=1}^{n} D_{\alpha}\left(\boldsymbol{A}^{\alpha \beta}(x) D_{\beta} \boldsymbol{u}\right)=0, \quad \boldsymbol{u}=\left(u^{1}, \ldots, u^{N}\right)^{T} .
$$

The adjoint system of (2.2) is given by

$$
L^{*} \boldsymbol{u}:=\sum_{\alpha, \beta=1}^{n} D_{\alpha}\left(\left(\boldsymbol{A}^{\alpha \beta}\right)^{*}(x) D_{\beta} \boldsymbol{u}\right)=0,
$$

where $\left(\boldsymbol{A}^{\alpha \beta}\right)^{*}=\left(\boldsymbol{A}^{\beta \alpha}\right)^{T}$, the transpose of $\boldsymbol{A}^{\beta \alpha}$.

Definition 2.3. We say that a system of $N$ equations on $\mathbb{R}^{n+1}$,

$$
u_{t}^{i}-\sum_{j=1}^{N} \sum_{\alpha, \beta=1}^{n} D_{x_{\alpha}}\left(A_{i j}^{\alpha \beta}(x) D_{x_{\beta}} u^{j}\right)=0 \quad(i=1, \ldots, N),
$$

is parabolic if the (time-independent) coefficients satisfy the uniform ellipticity condition. We often write the above system in a vector form:

$$
\boldsymbol{u}_{t}-L \boldsymbol{u}:=\boldsymbol{u}_{t}-\sum_{\alpha, \beta=1}^{n} D_{\alpha}\left(\boldsymbol{A}^{\alpha \beta}(x) D_{\beta} \boldsymbol{u}\right)=0 .
$$

The adjoint system of (2.4) is given by

$$
\boldsymbol{u}_{t}+L^{*} \boldsymbol{u}:=\boldsymbol{u}_{t}+\sum_{\alpha, \beta=1}^{n} D_{\alpha}\left(\left(\boldsymbol{A}^{\alpha \beta}\right)^{*}(x) D_{\beta} \boldsymbol{u}\right)=0,
$$

where $\left(\boldsymbol{A}^{\alpha \beta}\right)^{*}=\left(\boldsymbol{A}^{\beta \alpha}\right)^{T}$, the transpose of $\boldsymbol{A}^{\beta \alpha}$.

2.5. Weak solutions. In this article, the term "weak solution" is used in a rather abusive way. To avoid unnecessary technicalities, we may assume that all the coefficients involved are smooth so that all weak solutions are indeed classical solutions. However, this extra smoothness assumption will not be used quantitatively in our estimates. This is why we shall make clear the dependence of constants.

(1) We say that $\boldsymbol{u}$ is a weak solution of (2.2) in $\Omega \subset \mathbb{R}^{n}$ if $\boldsymbol{u}$ is a (classical) solution of (2.2) in $\Omega$ and $\boldsymbol{u}, D \boldsymbol{u} \in L^{2}(\Omega)$.

(2) We say that $\boldsymbol{u}$ is a weak solution of (2.4) in a cylinder $Q=\Omega \times(a, b) \subset \mathbb{R}^{n+1}$ if $\boldsymbol{u}$ is a (classical) solution of (2.2) in $Q$ and $\boldsymbol{u}, D \boldsymbol{u} \in L^{2}(Q), \boldsymbol{u}(\cdot, t) \in L^{2}(\Omega)$ for all $a \leq t \leq b$, and $\sup _{a \leq t \leq b}\|\boldsymbol{u}(\cdot, t)\|_{L^{2}(\Omega)}<\infty$.

2.6. Fundamental solution. By a fundamental solution (or fundamental matrix) $\boldsymbol{\Gamma}(x, t ; y)$ of the parabolic system (2.4) we mean an $N \times N$ matrix of functions defined for $t>0$ which, as a function of $(x, t)$, is a solution of (2.4) (i.e., each column is a solution of (2.4) $)$, and is such that

$$
\lim _{t \downarrow 0} \int_{\mathbb{R}^{n}} \boldsymbol{\Gamma}(x, t ; y) \boldsymbol{f}(y) d y=\boldsymbol{f}(x)
$$


for any bounded continuous function $\boldsymbol{f}=\left(f^{1}, \ldots, f^{N}\right)^{T}$, where $\boldsymbol{\Gamma}(x, t ; y) \boldsymbol{f}(y)$ denotes the usual matrix multiplication.

2.7. Notation for estimates. We employ the letter $C$ to denote a universal constant usually depending on the dimension and ellipticity constants. It should be understood that $C$ may vary from line to line. We sometimes write $C=C(\alpha, \beta, \ldots)$ to emphasize the dependence on the prescribed quantities $\alpha, \beta, \ldots$.

\subsection{Some preliminary results and known facts.}

Lemma 2.4 (Energy estimates). Let $\boldsymbol{u}$ be a weak solution of (2.4) in $Q_{R}=Q_{R}(X)$. Then for $0<r<R$, we have

$$
\sup _{t-r^{2} \leq s \leq t} \int_{Q_{r, s}}|\boldsymbol{u}(\cdot, s)|^{2}+\int_{Q_{r}}|D \boldsymbol{u}|^{2} \leq \frac{C}{(R-r)^{2}} \int_{Q_{R}}|\boldsymbol{u}|^{2} .
$$

Proof. See e.g., [14, Lemma 2.1, p. 139].

Lemma 2.5 (Parabolic Poincaré inequality). Let $\boldsymbol{u}$ be a weak solution of (2.4) in $Q_{R}=Q_{R}(X)$. Then there is some constant $C=C\left(n, M_{0}\right)$ such that

$$
\int_{Q_{R}}\left|\boldsymbol{u}-\overline{\boldsymbol{u}}_{Q_{R}}\right|^{2} \leq C R^{2} \int_{Q_{R}}|D \boldsymbol{u}|^{2} .
$$

Proof. See e.g., [19, Lemma 3].

Lemma 2.6. Let $Q_{2 R}=Q_{2 R}\left(X_{0}\right)$ be a cylinder in $\mathbb{R}^{n+1}$. Suppose $\boldsymbol{u} \in L^{2}\left(Q_{2 R}\right)$ and there are positive constants $\mu \leq 1$ and $M$ such that for any $X \in Q_{R}$ and any $r \in(0, R)$ we have

$$
\int_{Q_{r}(X)}\left|\boldsymbol{u}-\overline{\boldsymbol{u}}_{Q_{r}(X)}\right|^{2} \leq M^{2} r^{n+2+2 \mu} .
$$

Then $\boldsymbol{u}$ is Hölder continuous in $Q_{R}$ with the exponent $\mu$ and $[\boldsymbol{u}]_{C_{P}^{\mu}\left(Q_{R}\right)} \leq C(n, \mu) M$.

Proof. See e.g., [15, Lemma 4.3, p. 50].

Definition 2.7 (Local boundedness property). We say that the system (2.4) satisfies the local boundedness property for weak solutions if there is a constant $M$ such that all weak solutions $\boldsymbol{u}$ of (2.4) in $Q_{2 r}(X)$ satisfy the estimates

$$
\sup _{Q_{r}(X)}|\boldsymbol{u}| \leq M\left(\frac{1}{\left|Q_{2 r}\right|} \int_{Q_{2 r}(X)}|\boldsymbol{u}|^{2}\right)^{1 / 2} .
$$

Similarly, we say that the adjoint system (2.5) satisfies the local boundedness property if the corresponding estimates hold for weak solutions $\boldsymbol{u}$ of (2.5) in $Q_{2 r}^{*}(X)$.

Theorem 2.8 (Theorem 1.1, [12]). Assume that the system (2.4) and its adjoint system (2.5) satisfy the local boundedness property for weak solutions. Then the fundamental solution of the system (2.4) has an upper bound

$$
|\boldsymbol{\Gamma}(x, t ; y)|_{o p} \leq C_{0} t^{-n / 2} \exp \left(-\frac{k_{0}|x-y|^{2}}{t}\right),
$$

where $|\boldsymbol{\Gamma}(x, t ; y)|_{\text {op }}$ denotes the operator norm of the fundamental matrix $\boldsymbol{\Gamma}(x, t ; y)$. Here, $C_{0}=C_{0}\left(n, \nu_{0}, M_{0}, M\right)$ and $k_{0}=k_{0}\left(\nu_{0}, M_{0}\right)$. 


\section{MAIN RESULTS}

Definition 3.1. We say that an elliptic system (2.2) satisfies the Hölder estimates for weak solutions at every scale if there exist constants $\mu_{0}>0$ and $H_{0}$ such that all weak solutions $\boldsymbol{u}$ of the system in $B_{2 r}=B_{2 r}\left(x_{0}\right)$ satisfy the following estimates:

$$
[\boldsymbol{u}]_{C^{\mu_{0}\left(B_{r}\right)}} \leq H_{0} r^{-\left(n / 2+\mu_{0}\right)}\|\boldsymbol{u}\|_{L^{2}\left(B_{2 r}\right)} .
$$

Similarly, we say that a parabolic system (2.4) satisfies Hölder estimates for weak solutions at every scale if there exist constants $\mu_{1}>0$ and $H_{1}$ such that all weak solutions $\boldsymbol{u}$ of the system in $Q_{2 r}=Q_{2 r}\left(X_{0}\right)$ satisfy the following estimates:

$$
[\boldsymbol{u}]_{C_{P}^{\mu_{1}}\left(Q_{r}\right)} \leq H_{1} r^{-\left(n / 2+1+\mu_{1}\right)}\|\boldsymbol{u}\|_{L^{2}\left(Q_{2 r}\right)} .
$$

Remark 3.2. Elliptic systems with constant coefficients satisfy the above property, and in that case, the ellipticity condition (2.1) can be weakened and replaced by the Legendre-Hadamard condition. De Giorgi's theorem [7] states that the property is satisfied if $N=1$. The property is also satisfied if $n=2$ and it is due to Morrey (see Corollary [3.6). Some other examples include, for instance, a certain three dimensional elliptic system which was studied by Kang and the author in [13.

We shall prove the following main results in this paper:

Theorem 3.3. If an elliptic system (2.2) satisfies the Hölder estimates for weak solutions at every scale, then the corresponding parabolic system (2.4) with timeindependent coefficients also satisfies the Hölder estimates for weak solutions at every scale.

Theorem 3.4. Suppose that the elliptic system (2.2) and its adjoint system (2.3) defined on $\mathbb{R}^{n}$ both satisfy the Hölder estimates for weak solutions at every scale with constants $\mu_{0}, H_{0}$. Let $\boldsymbol{\Gamma}(x, t ; y)$ be the fundamental solution of the parabolic system (2.4) with the time-independent coefficients associated to the elliptic system (2.2). Then $\boldsymbol{\Gamma}(x, t ; y)$ has an upper bound

$$
|\boldsymbol{\Gamma}(x, t ; y)|_{o p} \leq C_{0} t^{-n / 2} \exp \left(-\frac{k_{0}|x-y|^{2}}{t}\right),
$$

where $C_{0}=C_{0}\left(n, \nu_{0}, M_{0}, \mu_{0}, H_{0}\right)$ and $k_{0}=k_{0}\left(\nu_{0}, M_{0}\right)$. Here, $|\boldsymbol{\Gamma}(x, t ; y)|_{\text {op }}$ denotes the operator norm of fundamental matrix $\boldsymbol{\Gamma}(x, t ; y)$.

Remark 3.5. We would like to point out that (3.3) is a global estimate. Especially, the bound (3.3) holds for all time $t>0$. Suppose that the elliptic system (2.2) and its adjoint system (2.3) enjoy the Hölder estimates for weak solutions up to a fixed scale $R_{0}$; that is, there is a number $R_{0}>0$ such that if $\boldsymbol{u}$ is a weak solution of either (2.2) or (2.3) in $B_{r}=B_{r}(x)$ with $0<r \leq R_{0}$, then $\boldsymbol{u}$ is Hölder continuous and satisfies

$$
[\boldsymbol{u}]_{C^{\mu_{0}\left(B_{r}\right)}} \leq H_{0} r^{-\left(n / 2+\mu_{0}\right)}\|\boldsymbol{u}\|_{L^{2}\left(B_{2 r}\right)} .
$$

Then, the statement regarding the bound (3.3) for the fundamental solution should be localized as follows: For any given $T>0$, there are constants $k_{0}=k_{0}\left(\nu_{0}, M_{0}\right)$ and $C_{0}=C_{0}\left(n, \nu_{0}, M_{0}, \mu_{0}, H_{0}, R_{0}, T\right)$ such that (3.3) holds for $0<t \leq T$.

Corollary 3.6. Let $\boldsymbol{\Gamma}(x, t ; y)$ be the fundamental solution of the parabolic system (2.4) with time-independent coefficients associated to an elliptic system (2.2) defined on $\mathbb{R}^{2}$. Then $\boldsymbol{\Gamma}(x, t ; y)$ has an upper bound (3.3) with the constants $C_{0}, k_{0}$ depending only on the ellipticity constants $\nu_{0}, M$. 
Proof. First, let us recall the well known theorem of Morrey which states that any two dimensional elliptic system (2.2) with bounded measurable coefficients satisfies the Hölder estimates for weak solutions at every scale, with the constants $\mu_{0}, H_{0}$ depending only on the ellipticity constants (see, [16, pp. 143-148]). Next, note that the ellipticity constants $\nu_{0}, M_{0}$ in (2.1) remain unchanged for $\tilde{A}_{i j}^{\alpha \beta}(x)=A_{j i}^{\beta \alpha}(x)$. Therefore, the corollary is an immediate consequence of Theorem 3.4 .

Remark 3.7. In fact, the converse of Theorem 2.8] is also true (see [12, Theorem 1.2]). Therefore, in order to extend the above corollary to the parabolic system with time-dependent coefficients, one needs to show that the system satisfies the local boundedness property for weak solutions. Unfortunately, we do not know whether it is true or not if the coefficients are allowed to depend on the time variable. If $n \geq 3$, it is not true in general, even for the time-independent coefficients case, since there is a famous counterexample due to De Giorgi (see [8]).

\section{Proof of the main Results}

\subsection{Some technical lemmas and proofs.}

Lemma 4.1. If $\boldsymbol{u}$ is a weak solution of the parabolic system with time-independent coefficients (2.4) in $Q_{R}=Q_{R}\left(X_{0}\right)$, then $\boldsymbol{u}_{t} \in L^{2}\left(Q_{r}\right)$ for $r<R$ and satisfies the estimates

$$
\left\|\boldsymbol{u}_{t}\right\|_{L^{2}\left(Q_{r}\right)} \leq C(R-r)^{-1}\|D \boldsymbol{u}\|_{L^{2}\left(Q_{R}\right)} .
$$

In particular, if $\boldsymbol{u}$ is a weak solution of (2.4) in $Q_{2 r}$, then the above estimates together with the energy estimates yield

$$
\left\|\boldsymbol{u}_{t}\right\|_{L^{2}\left(Q_{r}\right)} \leq C r^{-2}\|\boldsymbol{u}\|_{L^{2}\left(Q_{2 r}\right)} .
$$

Proof. We first note that if the coefficients are symmetric (i.e., $A_{i j}^{\alpha \beta}=A_{j i}^{\beta \alpha}$ ) this is a well known result; a proof for such a case is found, for example, in [14, pp. $172-181]$ or in [9. pp. 360-364]. However, the standard proof does not carry over to the nonsymmetric coefficients case and for that reason, we provide a self-contained proof here.

Fix positive numbers $\sigma, \tau$ such that $\sigma<\tau \leq R$. Let $\zeta$ be a smooth cut-off function such that $\zeta \equiv 1$ in $Q_{\sigma}$ vanishes near $\partial_{P} Q_{\tau}$ and satisfies

$$
0 \leq \zeta \leq 1 \quad \text { and } \quad\left|\zeta_{t}\right|+|D \zeta|^{2} \leq C(\tau-\sigma)^{-2}
$$

Note that on each slice $Q_{\tau, s}$ we have

$$
\begin{aligned}
0 & =\int_{Q_{\tau, s}}\left(\boldsymbol{u}_{t}-D_{\alpha}\left(A^{\alpha \beta} D_{\beta} \boldsymbol{u}\right)\right) \cdot \zeta^{2} \boldsymbol{u}_{t} \\
& =\int_{Q_{\tau, s}} \zeta^{2}\left|\boldsymbol{u}_{t}\right|^{2}+\int_{Q_{\tau, s}} \zeta^{2}\left\langle\boldsymbol{A}^{\alpha \beta} D_{\beta} \boldsymbol{u}, D_{\alpha} \boldsymbol{u}_{t}\right\rangle+\int_{Q_{\tau, s}} 2 \zeta\left\langle\boldsymbol{A}^{\alpha \beta} D_{\beta} \boldsymbol{u}, D_{\alpha} \zeta \boldsymbol{u}_{t}\right\rangle .
\end{aligned}
$$


Therefore, we find by using the Cauchy-Schwarz inequality that

$$
\begin{aligned}
\int_{Q_{\tau, s}} \zeta^{2}\left|\boldsymbol{u}_{t}\right|^{2} & \leq C \int_{Q_{\tau, s}} \zeta^{2}|D \boldsymbol{u}|\left|D \boldsymbol{u}_{t}\right|+C \int_{Q_{\tau, s}} \zeta|D \boldsymbol{u}||D \zeta|\left|\boldsymbol{u}_{t}\right| \\
& \leq \frac{\epsilon}{2} \int_{Q_{\tau, s}} \zeta^{2}\left|D \boldsymbol{u}_{t}\right|^{2}+\frac{C}{\epsilon} \int_{Q_{\tau, s}} \zeta^{2}|D \boldsymbol{u}|^{2}+C \int_{Q_{\tau, s}}|D \zeta|^{2}|D \boldsymbol{u}|^{2} \\
& +\frac{1}{2} \int_{Q_{\tau, s}} \zeta^{2}\left|\boldsymbol{u}_{t}\right|^{2} .
\end{aligned}
$$

Thus we have

$$
\int_{Q_{\tau}} \zeta^{2}\left|\boldsymbol{u}_{t}\right|^{2} \leq \epsilon \int_{Q_{\tau}} \zeta^{2}\left|D \boldsymbol{u}_{t}\right|^{2}+\frac{C}{\epsilon} \int_{Q_{\tau}} \zeta^{2}|D \boldsymbol{u}|^{2}+C \int_{Q_{\tau}}|D \zeta|^{2}|D \boldsymbol{u}|^{2} .
$$

Since $\boldsymbol{u}_{t}$ also satisfies (2.4), the energy estimates yield

$$
\int_{Q_{\tau}} \zeta^{2}\left|D \boldsymbol{u}_{t}\right|^{2} \leq \frac{C_{0}}{(\tau-\sigma)^{2}} \int_{Q_{\tau}}\left|\boldsymbol{u}_{t}\right|^{2}
$$

This is the part where we exploit the assumption that the coefficients are timeindependent. Combining (4.3) and (4.4), we have

$$
\int_{Q_{\sigma}}\left|\boldsymbol{u}_{t}\right|^{2} \leq \frac{C_{0} \epsilon}{(\tau-\sigma)^{2}} \int_{Q_{\tau}}\left|\boldsymbol{u}_{t}\right|^{2}+\frac{C}{\epsilon} \int_{Q_{\tau}}|D \boldsymbol{u}|^{2}+\frac{C}{(\tau-\sigma)^{2}} \int_{Q_{\tau}}|D \boldsymbol{u}|^{2} .
$$

If we set $\epsilon=(\tau-\sigma)^{2} / 2 C_{0}$, we finally obtain

$$
\int_{Q_{\sigma}}\left|\boldsymbol{u}_{t}\right|^{2} \leq \frac{1}{2} \int_{Q_{\tau}}\left|\boldsymbol{u}_{t}\right|^{2}+\frac{C}{(\tau-\sigma)^{2}} \int_{Q_{\tau}}|D \boldsymbol{u}|^{2} .
$$

Here, we emphasize that $C$ is a constant independent of $\sigma, \tau$. Then by a standard iteration argument (see e.g., [11, Lemma 3.1, p. 161]), we have

$$
\int_{Q_{r}}\left|\boldsymbol{u}_{t}\right|^{2} \leq \frac{C}{(R-r)^{2}} \int_{Q_{R}}|D \boldsymbol{u}|^{2} \quad \text { for } 0<r<R .
$$

The proof is complete.

Lemma 4.2. If $\boldsymbol{u}$ is a weak solution of the parabolic system with time-independent coefficients (2.4) in $Q_{2 r}=Q_{2 r}\left(X_{0}\right)$, then $D \boldsymbol{u}(\cdot, s), \boldsymbol{u}_{t}(\cdot, s) \in L^{2}\left(Q_{r, s}\right)$ for all $s \in$ $\left[t_{0}-r^{2}, t_{0}\right]$, and satisfies the following estimates uniformly in $s \in\left[t_{0}-r^{2}, t_{0}\right]$ :

$$
\begin{aligned}
\|D \boldsymbol{u}(\cdot, s)\|_{L^{2}\left(Q_{r, s}\right)} & \leq C r^{-2}\|\boldsymbol{u}\|_{L^{2}\left(Q_{2 r}\right)}, \\
\left\|\boldsymbol{u}_{t}(\cdot, s)\right\|_{L^{2}\left(Q_{r, s}\right)} & \leq C r^{-3}\|\boldsymbol{u}\|_{L^{2}\left(Q_{2 r}\right)} .
\end{aligned}
$$

Proof. By the energy estimates applied to $\boldsymbol{u}_{t}$ we obtain

$$
\sup _{t_{0}-r^{2} \leq s \leq t_{0}} \int_{Q_{r, s}}\left|\boldsymbol{u}_{t}(\cdot, s)\right|^{2} \leq \frac{C}{r^{2}} \int_{Q_{3 r / 2}}\left|\boldsymbol{u}_{t}\right|^{2} .
$$

On the other hand, the estimates (4.5) and the energy estimates (this time, applied to $\boldsymbol{u}$ itself) yield

$$
\int_{Q_{3 r / 2}}\left|\boldsymbol{u}_{t}\right|^{2} \leq \frac{C}{r^{2}} \int_{Q_{7 r / 4}}|D \boldsymbol{u}|^{2} \leq \frac{C}{r^{4}} \int_{Q_{2 r}}|\boldsymbol{u}|^{2} .
$$

Combining (4.8) and (4.9) together, we have the estimates (4.7). 
Next, assume that $\boldsymbol{u}$ is a weak solution of (2.4) in $Q_{4 r}=Q_{4 r}\left(X_{0}\right)$. Let $\zeta$ be a smooth cut-off function such that $\zeta \equiv 1$ in $Q_{r}$ vanishes near $\partial_{P} Q_{2 r}$, and satisfies

$$
0 \leq \zeta \leq 1 \quad \text { and } \quad\left|\zeta_{t}\right|+|D \zeta|^{2} \leq C r^{-2}
$$

Note that on each slice $Q_{2 r, s}$, we have

$$
\begin{aligned}
0 & =\int_{Q_{2 r, s}}\left(\boldsymbol{u}_{t}-D_{\alpha}\left(\boldsymbol{A}^{\alpha \beta} D_{\beta} \boldsymbol{u}\right)\right) \cdot \zeta^{2} \boldsymbol{u} \\
& =\int_{Q_{2 r, s}} \zeta^{2} \boldsymbol{u}_{t} \cdot \boldsymbol{u}+\int_{Q_{2 r, s}} \zeta^{2}\left\langle\boldsymbol{A}^{\alpha \beta} D_{\beta} \boldsymbol{u}, D_{\alpha} \boldsymbol{u}\right\rangle+2 \zeta\left\langle\boldsymbol{A}^{\alpha \beta} D_{\beta} \boldsymbol{u}, D_{\alpha} \zeta \boldsymbol{u}\right\rangle .
\end{aligned}
$$

Using the ellipticity condition and the Cauchy-Schwarz inequality, we find

$$
\begin{aligned}
\nu_{0} \int_{Q_{2 r, s}} \zeta^{2}|D \boldsymbol{u}|^{2} & \leq \int_{Q_{2 r, s}} \zeta^{2}\left|\boldsymbol{u}_{t}\right||\boldsymbol{u}|+C \int_{Q_{2 r, s}} \zeta|D \boldsymbol{u}||D \zeta||\boldsymbol{u}| \\
& \leq \frac{\epsilon \nu_{0}}{2} \int_{Q_{2 r, s}} \zeta^{2}\left|\boldsymbol{u}_{t}\right|^{2}+\frac{C}{\epsilon} \int_{Q_{2 r, s}} \zeta^{2}|\boldsymbol{u}|^{2}+C \int_{Q_{2 r, s}}|D \zeta|^{2}|\boldsymbol{u}|^{2} \\
& +\frac{\nu_{0}}{2} \int_{Q_{2 r, s}} \zeta^{2}|D \boldsymbol{u}|^{2}
\end{aligned}
$$

Then by (4.10), (4.7), and the energy estimates, for all $s \in\left[t_{0}-r^{2}, t_{0}\right]$, we have

$$
\begin{aligned}
\int_{Q_{r, s}}|D \boldsymbol{u}|^{2} & \leq \epsilon \int_{Q_{2 r, s}}\left|\boldsymbol{u}_{t}\right|^{2}+\frac{C}{\epsilon} \int_{Q_{2 r, s}}|\boldsymbol{u}|^{2}+\frac{C}{r^{2}} \int_{Q_{2 r, s}}|\boldsymbol{u}|^{2} \\
& \leq \frac{C \epsilon}{r^{6}} \int_{Q_{4 r}}|\boldsymbol{u}|^{2}+\frac{C}{\epsilon r^{2}} \int_{Q_{4 r}}|\boldsymbol{u}|^{2}+\frac{C}{r^{4}} \int_{Q_{4 r}}|\boldsymbol{u}|^{2} .
\end{aligned}
$$

If we set $\epsilon=r^{2}$, then the above estimates (4.11) now become

$$
\int_{Q_{r, s}}|D \boldsymbol{u}|^{2} \leq \frac{C}{r^{4}} \int_{Q_{4 r}}|\boldsymbol{u}|^{2}
$$

from which the estimate (4.6) follows by a well known covering argument.

Lemma 4.3. Assume that the elliptic system (2.2) satisfies the Hölder estimates for weak solutions at every scale with constants $\mu_{0}, H_{0}$. Let $\boldsymbol{u}$ be a weak solution of the inhomogeneous elliptic system

$$
D_{\alpha}\left(\boldsymbol{A}^{\alpha \beta}(x) D_{\beta} \boldsymbol{u}\right)=\boldsymbol{f} \quad \text { in } \quad B_{2}=B_{2}\left(x_{0}\right),
$$

where $\boldsymbol{f}$ belongs to the Morrey space $M^{2, \lambda}\left(B_{2}\right)$ with $\lambda \geq 0$. Then, for any $\gamma \geq 0$ with $\gamma<\gamma_{0}=\min \left(\lambda+4, n+2 \mu_{0}\right)$ (we may take $\gamma=\gamma_{0}$ if $\gamma_{0}<n$ ) there exists $C=C\left(n, \nu_{0}, M_{0}, \mu_{0}, H_{0}, \lambda, \gamma\right)$ such that $\boldsymbol{u}$ satisfies the following local estimates:

$$
\int_{B_{r}(x)}|D \boldsymbol{u}|^{2} \leq C\left(r^{\gamma-2} \int_{B_{2}}|D \boldsymbol{u}|^{2}+r^{\gamma-2}\|\boldsymbol{f}\|_{M^{2, \lambda}\left(B_{2}\right)}^{2}\right)
$$

uniformly for all $x \in B_{1}=B_{1}\left(x_{0}\right)$ and $0<r \leq 1$. Moreover, if $\gamma<n$, then $\boldsymbol{u}$ belongs to the Morrey space $M^{2, \gamma}\left(B_{1}\right)$ and

$$
\|\boldsymbol{u}\|_{M^{2, \gamma}\left(B_{1}\right)} \leq C\left(\|\boldsymbol{u}\|_{L^{2}\left(B_{2}\right)}+\|D \boldsymbol{u}\|_{L^{2}\left(B_{2}\right)}+\|\boldsymbol{f}\|_{M^{2, \lambda}\left(B_{2}\right)}\right) .
$$


Proof. First, we note that the property (3.1) implies that for all $0<\rho<r$ and $x \in \mathbb{R}^{n}$, we have

$$
\int_{B_{\rho}(x)}|D \boldsymbol{u}|^{2} \leq C \cdot H_{0}\left(\frac{\rho}{r}\right)^{n-2+2 \mu_{0}} \int_{B_{r}(x)}|D \boldsymbol{u}|^{2} .
$$

In light of the above observation, the estimate (4.13) is quite standard and is found, for example, in [11, Chapter 3]. Then, by the Poincaré inequality we have

$$
\int_{B_{r}(x)}\left|\boldsymbol{u}-\overline{\boldsymbol{u}}_{B_{r}(x)}\right|^{2} \leq C r^{\gamma}\left(\|D \boldsymbol{u}\|_{L^{2}\left(B_{2}\right)}^{2}+\|\boldsymbol{f}\|_{M^{2, \lambda}\left(B_{2}\right)}^{2}\right)
$$

uniformly for all $x \in B_{1}=B_{1}(0)$ and $0<r \leq 1$. It is well known that if $\gamma<n$, then the estimates (4.15) yield (4.14) (see e.g., [11, Chapter 3]).

4.2. Proof of Theorem 3.3. Let $\boldsymbol{u}$ be a weak solution of (2.4) in a cylinder $Q_{4}=Q_{4}(0)$. We rewrite (2.4) as $L \boldsymbol{u}=\boldsymbol{u}_{t}$. By Lemma 4.2, we find that $\boldsymbol{u}_{t}(\cdot, s)$ is in $L^{2}\left(Q_{2, s}\right)$ and satisfies

$$
\left\|\boldsymbol{u}_{t}(\cdot, s)\right\|_{L^{2}\left(Q_{2, s}\right)} \leq C\|\boldsymbol{u}\|_{L^{2}\left(Q_{4}\right)} \quad \text { for all }-4 \leq s \leq 0 .
$$

Therefore, we may apply Lemma 4.3 with $\boldsymbol{f}=\boldsymbol{u}_{t}$ and $\lambda=0$, and then apply Lemma 4.2 to find that for all $x \in B_{1}(0)$ and $0<r \leq 1$, we have

$$
\begin{aligned}
\int_{B_{r}(x)}|D \boldsymbol{u}(\cdot, s)|^{2} & \leq C r^{\gamma-2}\left(\|D \boldsymbol{u}(\cdot, s)\|_{L^{2}\left(Q_{2, s}\right)}^{2}+\left\|\boldsymbol{u}_{t}(\cdot, s)\right\|_{L^{2}\left(Q_{2, s}\right)}^{2}\right) \\
& \leq C r^{\gamma-2}\|\boldsymbol{u}\|_{L^{2}\left(Q_{4}\right)}^{2} \quad \text { uniformly in } s \in[-4,0]
\end{aligned}
$$

for all $\gamma<\min \left(4, n+2 \mu_{0}\right)$.

By Lemma 2.5 and then by (4.16) we find that for all $X=(x, t) \in Q_{1}$ and $r \leq 1$

$$
\begin{aligned}
\int_{Q_{r}(X)}\left|\boldsymbol{u}-\overline{\boldsymbol{u}}_{Q_{r}(X)}\right|^{2} & =C r^{2} \int_{t-r^{2}}^{t} \int_{B_{r}(x)}|D \boldsymbol{u}(y, s)|^{2} d y d s \\
& \leq C r^{2+\gamma}\|\boldsymbol{u}\|_{L^{2}\left(Q_{4}\right)}^{2} .
\end{aligned}
$$

Note that if $n \leq 3$, then we may write $\gamma=n+2 \mu$ for some $\mu>0$. In that case, (4.17) now reads

$$
\int_{Q_{r}(X)}\left|\boldsymbol{u}-\overline{\boldsymbol{u}}_{Q_{r}(X)}\right|^{2} \leq C r^{n+2+2 \mu}\|\boldsymbol{u}\|_{L^{2}\left(Q_{4}\right)}^{2}
$$

for all $X \in Q_{1}$ and $r \leq 1$. Therefore, if $n \leq 3$, then Lemma 2.6 yields the estimates

$$
[\boldsymbol{u}]_{C_{P}^{\mu}\left(Q_{1 / 2}\right)} \leq C\|\boldsymbol{u}\|_{L^{2}\left(Q_{4}\right)} .
$$

We have thus shown that in the case when $n \leq 3$, any weak solution $\boldsymbol{u}$ of (2.4) in a cylinder $Q_{4}=Q_{4}(0)$ satisfies the above a priori estimates (4.19) provided that the associated elliptic system satisfies the Hölder estimates for weak solutions at every scale. The general case is recovered as follows. For given $X_{0}=\left(x_{0}, t_{0}\right)$ and $r>0$, let us consider the new system

$$
\boldsymbol{u}_{t}-\tilde{L} \boldsymbol{u}:=\boldsymbol{u}_{t}-\sum_{\alpha, \beta=1}^{n} D_{\alpha}\left(\tilde{\boldsymbol{A}}^{\alpha \beta}(x) D_{\beta} \boldsymbol{u}\right)=0,
$$

where $\tilde{\boldsymbol{A}}^{\alpha \beta}(x)=\boldsymbol{A}^{\alpha \beta}\left(x_{0}+r x\right)$. Note that the associated elliptic system $\tilde{L} \boldsymbol{u}=0$ also satisfies the Hölder estimates for weak solutions at every scale. Moreover, the ellipticity constants $\nu_{0}, M_{0}$ remain the same for the new coefficients $\tilde{\boldsymbol{A}}^{\alpha \beta}$. Let $\boldsymbol{u}$ be 
a weak solution of (2.4) in $Q_{4 r}\left(X_{0}\right)$. Then $\tilde{\boldsymbol{u}}(X)=\tilde{\boldsymbol{u}}(x, t):=\boldsymbol{u}\left(x_{0}+r x, t_{0}+r^{2} t\right)$ is a weak solution of (4.20) in $Q_{4}(0)$ and thus $\tilde{\boldsymbol{u}}$ satisfies the estimate (4.19). By rescaling back to $Q_{4 r}\left(X_{0}\right)$, the estimate (4.19) becomes

$$
[\boldsymbol{u}]_{C_{P}^{\mu}\left(Q_{r / 2}\right)} \leq C r^{-(n / 2+1+\mu)}\|\boldsymbol{u}\|_{L^{2}\left(Q_{4 r}\right)} .
$$

Thus, when $n \leq 3$, the theorem now follows from a well known covering argument.

In the case when $n \geq 4$, we invoke a bootstrap argument. For the sake of simplicity, let us momentarily assume that $4 \leq n \leq 7$. Let $\boldsymbol{u}$ be a weak solution of (2.4) in $Q_{8}=Q_{8}(0)$. Let us fix $X_{0}=\left(x_{0}, t_{0}\right) \in Q_{2}(0)$ and observe that $\boldsymbol{u}_{t}$ also satisfies the system (2.4) in $Q_{4}\left(X_{0}\right)$. Thus, by a similar argument that led to (4.16), we find that for all $x \in B_{1}\left(x_{0}\right)$ and $0<r \leq 1$ we have

$$
\int_{B_{r}(x)}\left|D \boldsymbol{u}_{t}(\cdot, s)\right|^{2} \leq C r^{\gamma-2}\left\|\boldsymbol{u}_{t}\right\|_{L^{2}\left(Q_{4}\left(X_{0}\right)\right)}^{2} \quad \text { uniformly in } s \in\left[t_{0}-4, t_{0}\right],
$$

for all $\gamma<4$ (we may take $\gamma=4$ if $n>4$ ). Then, by (4.14) in Lemma 4.3, Lemma 4.1 and Lemma 4.2 we conclude that

$$
\left\|\boldsymbol{u}_{t}(\cdot, s)\right\|_{M^{2, \gamma}\left(B_{1}\left(x_{0}\right)\right)} \leq C\|\boldsymbol{u}\|_{L^{2}\left(Q_{8}(0)\right)} \quad \text { for all } s \in\left[t_{0}-4, t_{0}\right] .
$$

Since the above estimate (4.23) hold for all $X_{0}=\left(x_{0}, t_{0}\right) \in Q_{2}(0)$, we find that, in particular, $\boldsymbol{u}_{t}(\cdot, s)$ belongs to $M^{2, \gamma}\left(B_{2}(0)\right)$ for all $-4 \leq s \leq 0$, and satisfies

$$
\left\|\boldsymbol{u}_{t}(\cdot, s)\right\|_{M^{2, \gamma}\left(B_{2}(0)\right)} \leq C\|\boldsymbol{u}\|_{L^{2}\left(Q_{8}(0)\right)} \quad \text { for all } s \in[-4,0],
$$

where we also used (4.7) of Lemma 4.2 .

The above estimates (4.24) for $\boldsymbol{u}_{t}$ now allows us to invoke Lemma 4.3 with $\boldsymbol{f}=\boldsymbol{u}_{t}$ and $\lambda=\gamma$. Then, by Lemma 4.3 and Lemma 4.2. we find that for all $x \in B_{1}(0)$ and $0<r \leq 1$, we have

$$
\int_{B_{r}(x)}|D \boldsymbol{u}(\cdot, s)|^{2} \leq C r^{\bar{\gamma}-2}\|\boldsymbol{u}\|_{L^{2}\left(Q_{8}(0)\right)}^{2} \quad \text { uniformly in } s \in[-4,0]
$$

for all $\bar{\gamma}<\min \left(\gamma+4, n+2 \mu_{0}\right)$. Since we assume that $n \leq 7$, we may write $\bar{\gamma}=n+2 \bar{\mu}$ for some $\bar{\mu}>0$. By the exactly same argument we used in the case when $n \leq 3$, we derive the estimates

$$
[\boldsymbol{u}]_{C_{P}^{\mu}\left(Q_{1 / 2}\right)} \leq C\|\boldsymbol{u}\|_{L^{2}\left(Q_{8}\right)}
$$

and the theorem follows as before.

Finally, if $n \geq 8$, we repeat the above process; if $\boldsymbol{u}$ is a weak solution of (2.4) in $Q_{16}(0)$, then $\boldsymbol{u}_{t}(\cdot, s)$ is in $M^{2, \gamma}\left(B_{1}(0)\right)$ for all $\gamma<8$, and so on. The process cannot go on indefinitely, and it stops in $k=[n / 4]+1$ steps. The proof is complete.

4.3. Proof of Theorem 3.4. The proof is based on Theorem 2.8, the proof of which, in turn, is found in [12]. By Theorem [2.8, we only need to establish the local boundedness property for weak solutions of the parabolic system (2.4) and for those of its adjoint system (2.5).

From the hypothesis that the elliptic system (2.2) satisfies the Hölder estimates for weak solutions at every scale, we find, by Theorem 3.3. that the parabolic system (2.4) with the associated time-independent coefficients also satisfies the Hölder estimates for weak solutions at every scale; that is, there exist some constants $\mu>0$ and $C$, depending on the prescribed quantities, such that if $\boldsymbol{u}$ is a weak solution of (2.4) in $Q_{4 r}(X)$, then it satisfies the estimates

$$
[\boldsymbol{u}]_{C_{P}^{\mu}\left(Q_{2 r}\right)} \leq C r^{-(n / 2+1+\mu)}\|\boldsymbol{u}\|_{L^{2}\left(Q_{4 r}\right)} .
$$


Let us fix $Y \in Q_{r}=Q_{r}(X)$. Then, for all $Z \in Q_{r}(Y) \subset Q_{2 r}(X)$, we have

$$
|\boldsymbol{u}(Y)| \leq|\boldsymbol{u}(Z)|+d_{P}(Y, Z)^{\mu} \cdot[\boldsymbol{u}]_{C_{P}^{\mu}\left(Q_{2 r}\right)} \leq|\boldsymbol{u}(Z)|+C r^{-(n / 2+1)}\|\boldsymbol{u}\|_{L^{2}\left(Q_{4 r}\right)} .
$$

By averaging (4.25) over $Q_{r}(Y)$ with respect to $Z$, we derive (note $\left|Q_{r}\right|=C r^{n+2}$ )

$$
|\boldsymbol{u}(Y)| \leq C r^{-(n+2)}\|\boldsymbol{u}\|_{L^{1}\left(Q_{r}(Y)\right)}+C r^{-(n / 2+1)}\|\boldsymbol{u}\|_{L^{2}\left(Q_{4 r}\right)} .
$$

Since $Y \in Q_{r}$ is arbitrary, we find, by Hölder's inequality, that $\boldsymbol{u}$ satisfies

$$
\|\boldsymbol{u}\|_{L^{\infty}\left(Q_{r}\right)} \leq C r^{-(n / 2+1)}\|\boldsymbol{u}\|_{L^{2}\left(Q_{4 r}\right)}
$$

for some constant $C=C\left(n, \nu_{0}, M_{0}, \mu_{0}, H_{0}\right)$.

To finish the proof, we also need to show that if $\boldsymbol{u}$ is a weak solution of the adjoint system (2.5) in $Q_{4 r}^{*}=Q_{4 r}^{*}(X)$, then it satisfies the local boundedness property

$$
\|\boldsymbol{u}\|_{L^{\infty}\left(Q_{r}^{*}\right)} \leq C r^{-(n / 2+1)}\|\boldsymbol{u}\|_{L^{2}\left(Q_{4 r}^{*}\right)} .
$$

The verification of (4.26) requires only a slight modification of the previous arguments (mostly, one needs to replace $Q_{r}$ by $Q_{r}^{*}$, and so on), but it is rather routine and so we skip the details.

\section{REFERENCES}

1. Aronson, D. G. Bounds for the fundamental solution of a parabolic equation. Bull. Amer. Math. Soc. 73 (1967) 890-896. MR0217444(36:534)

2. Auscher, P. Regularity theorems and heat kernel for elliptic operators. J. London Math. Soc. (2) 54 (1996), no. 2, 284-296. MR1405056 (97f:35034)

3. Auscher, P.; McIntosh, A.; Tchamitchian, Ph. Heat kernels of second order complex elliptic operators and applications. J. Funct. Anal. 152 (1998), no. 1, 22-73. MR.1600066 (99e:47062)

4. Auscher, P.; Qafsaoui, M. Equivalence between regularity theorems and heat kernel estimates for higher order elliptic operators and systems under divergence form. J. Funct. Anal. $\mathbf{1 7 7}$ (2000), no. 2, 310-364. MR1795955 (2001j:35057)

5. Auscher, P.; Tchamitchian, Ph. Square root problem for divergence operators and related topics. Astérisque No. 249 (1998).

6. Davies, E. B. Explicit constants for Gaussian upper bounds on heat kernels, Amer. J. Math. 109 (1987), no. 2, 319-333. MR882426 (88g:58174)

7. De Giorgi, E. Sulla differenziabilità e l'analiticità delle estremali degli integrali multipli regolari. (Italian) Mem. Accad. Sci. Torino. Cl. Sci. Fis. Mat. Nat. (3) 3 (1957), 25-43. MR0093649 $(20: 172)$

8. De Giorgi, E. Un esempio di estremali discontinue per un problema variazionale di tipo ellittico. (Italian) Boll. Un. Mat. Ital. (4) 1 (1968), 135-137. MR0227827 (37:3411)

9. Evans, L. C. Partial differential equations. American Mathematical Society, Providence, RI, 1998. MR 1625845 (99e:35001)

10. Fabes, E. B.; Stroock, D. W. A new proof of Moser's parabolic Harnack inequality using the old ideas of Nash. Arch. Rational Mech. Anal. 96 (1986), no. 4, 327-338. MR855753 (88b:35037)

11. Giaquinta, M. Multiple integrals in the calculus of variations and nonlinear elliptic systems, Princeton University Press, Princeton, NJ, 1983. MR717034 (86b:49003)

12. Hofmann, S.; Kim, S. Gaussian estimates for fundamental solutions to certain parabolic systems. Publ. Mat. 48 (2004), 481-496. MR2091016 (2005g:35004)

13. Kang, K.; Kim, S. On the Hölder continuity of solutions of a certain system related to Maxwell's equations. SIAM J. Math. Anal. 34 (2002), no. 1, 87-100 (electronic). MR1950827 (2004h:35213)

14. Ladyženskaja, O. A.; Solonnikov, V. A.; Ural'ceva, N. N. Linear and quasilinear equations of parabolic type. American Mathematical Society, Providence, RI, 1967. MR0241822 (39:3159b)

15. Lieberman, G. M. Second order parabolic differential equations. World Scientific Publishing Co., Inc., River Edge, NJ, 1996. MR.1465184 (98k:35003)

16. Morrey, C. B., Jr. Multiple integrals in the calculus of variations. Springer-Verlag New York, Inc., New York, 1966. MR0202511 (34:2380) 
17. Moser, J. A Harnack inequality for parabolic differential equations. Comm. Pure Appl. Math. 17 (1964) 101-134; Correction: Comm. Pure Appl. Math. 20 (1967), 231-236. MR0159139 $(28: 2357)$

18. Nash, J. Continuity of solutions of parabolic and elliptic equations. Amer. J. Math. 80 (1958), 931-954. MR0100158 (20:6592)

19. Struwe, M. On the Hölder continuity of bounded weak solutions of quasilinear parabolic systems. Manuscripta Math. 35 (1981), no. 1-2, 125-145. MR627929 (83c:35022)

Department of Mathematics, Yonsei University, 262 Seongsanno, Seodaemun-gu, SEOUl 120-749, Korea

E-mail address: kimseick@yonsei.ac.kr 